\title{
Measuring overuse of continuous pulse oximetry in bronchiolitis and developing strategies for large-scale deimplementation: study protocol for a feasibility trial
}

Irit R. Rasooly ${ }^{1}$, Rinad S. Beidas ${ }^{2,3,4}$, Courtney Benjamin Wolk²,4, Frances Barg ${ }^{5}$, Christopher P. Landrigan ${ }^{6,7,8}$, Amanda Schondelmeyer ${ }^{9,10,11}$, Patrick W. Brady ${ }^{9,10,11}$, Lisa M. McLeod ${ }^{12,13}$, Christopher P. Bonafide ${ }^{1,4,14^{*}}$ and for the Pediatric Research in Inpatient Settings (PRIS) Network

\begin{abstract}
Background: Deimplementation, the systematic elimination of low-value practices, has emerged as an important focus within implementation science. Bronchiolitis is the leading cause of infant hospitalization. Among stable inpatients with bronchiolitis who do not require supplemental oxygen, continuous pulse oximetry monitoring is recognized as an overused, low-value practice in pediatric hospital medicine. There is strong scientific evidence and practice guideline support for limiting pulse oximetry monitoring of stable children with bronchiolitis who do not require supplemental oxygen, yet the practice remains common. This study aims to (1) characterize the extent of this overuse in hospitals located in the USA and Canada, (2) identify barriers and facilitators of successful deimplementation of continuous pulse oximetry monitoring in bronchiolitis, and (3) develop consensus strategies for large-scale deimplementation. In addition to identifying feasible strategies for deimplementation, this study will test the feasibility of data collection approaches to be employed in a large-scale deimplementation trial.

Methods: This multicenter study will be performed in approximately 38 hospitals in the Pediatric Research in Inpatient Settings Network. In Aim 1, we will determine the rate of overuse within each hospital by performing repeated crosssectional observational sampling of continuous pulse oximetry monitoring of stable bronchiolitis patients age 8 weeks through 23 months who do not require supplemental oxygen. In Aim 2, we will use the Consolidated Framework for Implementation Research (CFIR) as a framework for semi-structured interviews with key stakeholders (physician, nurse, respiratory therapist, administrator, and parent) at the highest- and lowest-overuse hospitals to understand barriers and facilitators of continuous pulse oximetry monitoring deimplementation. In Aim 3, we will use a theory-based causal model to match the identified barriers and facilitators to potential strategies for deimplementation. Candidate strategies will be discussed with a panel of stakeholders from hospitals with high rates of overuse to assess feasibility and acceptability. A questionnaire ranking strategies based on feasibility, acceptability, and impact will be administered to a broader group of stakeholders to arrive at consensus about promising strategies for large-scale deimplementation to be tested in a subsequent trial.

(Continued on next page)
\end{abstract}

\footnotetext{
* Correspondence: bonafide@email.chop.edu

'Section of Hospital Medicine, Children's Hospital of Philadelphia, Buerger

Center for Advanced Pediatric Care, 3500 Civic Center Blvd., 11th Floor,

Philadelphia, PA, USA

${ }^{4}$ Leonard Davis Institute of Health Economics, University of Pennsylvania,

3535 Market Street, Suite 3006, Philadelphia, PA, USA

Full list of author information is available at the end of the article
}

(c) The Author(s). 2019 Open Access This article is distributed under the terms of the Creative Commons Attribution 4.0 International License (http://creativecommons.org/licenses/by/4.0/), which permits unrestricted use, distribution, and reproduction in any medium, provided you give appropriate credit to the original author(s) and the source, provide a link to the Creative Commons license, and indicate if changes were made. The Creative Commons Public Domain Dedication waiver (http://creativecommons.org/publicdomain/zero/1.0/) applies to the data made available in this article, unless otherwise stated. 
(Continued from previous page)

Discussion: Effective strategies for deimplementing continuous pulse oximetry monitoring of stable patients with bronchiolitis have not been well characterized. The findings of this study will provide further understanding of factors that facilitate deimplementation in pediatric hospital settings and provide pilot and feasibility data to inform a trial of large-scale deimplementation of this overused practice.

Trial registration: Not applicable. This study does not meet the World Health Organization definition of a clinical trial.

Keywords: Bronchiolitis, Overuse, Deimplementation, Implementation science, Pediatric hospital medicine

\section{Background}

Deimplementation, the systematic, structured reduction or elimination of low-value practices [1-3], has emerged as a distinct focus within the field of implementation science. Reduction of overuse, the provision of unnecessary services for which the potential for harm outweighs the potential for benefit $[4,5]$, is a key application of deimplementation. In pediatrics, research on overuse has been largely descriptive $[6,7]$. While numerous candidate conditions for deimplementation have been identified [8-15], efforts to curb overuse have been limited. Application of rigorous implementation science methods to the development and evaluation of strategies for deimplementation is an essential next step to reducing pediatric overuse practices.

Continuous pulse oximetry $\left(\mathrm{SpO}_{2}\right)$ monitoring in bronchiolitis is an important area of overuse in pediatric hospital medicine. Bronchiolitis is the leading cause of infant hospitalization with over 100,000 annual admissions at a cost of over $\$ 1.7$ billion $[7,16]$. Hospital treatment is primarily supportive [17], focused on optimizing hydration and treating hypoxemia, and often includes continuous $\mathrm{SpO}_{2}$ monitoring. Though there is strong evidence to suggest continuous $\mathrm{SpO}_{2}$ monitoring stable patients with bronchiolitis is unnecessary and may even pose risk, the practice remains widespread. While formal study of the barriers to deimplementing this practice have not yet been undertaken, we speculate that potential reasons for the practice continuing include perceptions of a higher degree of safety conferred when patients are monitored, entrenched practices and customs to monitor patients, and clinicians' perceptions that families prefer that their children be monitored.

Outpatient studies of infants with bronchiolitis well enough to be discharged home from the emergency department without admission demonstrate that transient hypoxemia associated with bronchiolitis is common and likely of little clinical consequence [18]. Conversely, continuous monitoring has been associated with increased rates of admission, longer length of stay, more treatment with supplemental oxygen, and higher hospital cost, without improving outcomes $[19,20]$. More generally, from a system level perspective, continuous $\mathrm{SpO}_{2}$ monitoring overuse incurs the potential hazards of alarm fatigue. Alarm fatigue is a well-described phenomenon in which exposure to high rates of false alarms results in desensitization, leading to ignored or slowed responses to all alarms [21-24].

The safety of reducing/eliminating continuous pulse oximetry of stable patients with bronchiolitis has been demonstrated in quality improvement initiatives [25] and a randomized controlled trial [19]. Both the American Academy of Pediatrics [17] and the Society of Hospital Medicine Choosing Wisely in Pediatric Hospital Medicine workgroup [8] support limiting monitoring of children with bronchiolitis. The American Academy of Pediatrics Bronchiolitis Clinical Practice Guideline states that clinicians may choose not to use continuous $\mathrm{SpO}_{2}$ monitoring for children with bronchiolitis [17]. Choosing Wisely Guidelines state more firmly that clinicians should not use continuous $\mathrm{SpO}_{2}$ monitoring in children with acute respiratory illness unless they are receiving supplemental oxygen [8].

Despite the guidelines, the practice of using continuous $\mathrm{SpO}_{2}$ monitoring in stable bronchiolitis patients of supplemental oxygen remains common. A community hospital-based quality improvement collaborative reported post-intervention rates of continuous monitoring approaching $50 \%$, with marked variability between hospitals [26]. In order to develop a successful and broadly accepted intervention to reduce this potentially harmful practice, it is important to fully understand the barriers, facilitators, and contextual factors influencing (and impeding) successful deimplementation.

\section{Specific aims}

The specific aims of this study are:

(1) To measure baseline $\mathrm{SpO}_{2}$ monitoring overuse rates in children with bronchiolitis not requiring supplemental oxygen in 38 Pediatric Research in Inpatient Settings Network hospitals.

(2) To identify barriers and facilitators to deimplementing $\mathrm{SpO}_{2}$ monitoring in children with bronchiolitis by conducting semi-structured interviews with key stakeholders at the highestand lowest-overuse hospitals.

(3) Informed by contextual analysis, develop a multifaceted deimplementation strategy for $\mathrm{SpO}_{2}$ 
monitoring overuse in children with bronchiolitis that will serve as the intervention to be tested in a subsequent clinical trial.

This study will be one of the first comprehensive evaluations of an overused practice in pediatric hospital medicine, providing insight into the state of overuse, the variation in overuse across hospitals, and the factors that contribute to overuse in a wide variety of hospital settings. Applying rigorous and reproducible methods to the study of deimplementation practices and obtaining a deep, contextual understanding of barriers and facilitators to deimplementation are essential for identifying strategies capable of producing large-scale, sustained reductions in overuse of this widespread practice. Specifically, contextual analysis will be used in the following ways: (1) in Aim 2, the organizational and social contexts relevant to monitoring overuse and its deimplementation will be queried and analyzed along with the barriers and facilitators that we seek to identify, and (2) in Aim 3, we will build upon the knowledge of organizational and social contexts in order to design a strategy or menu of strategies that can be tailored, if appropriate, to different organizational and social contexts.

Understanding why current pulse oximetry monitoring recommendations are (or are not) adhered to is essential to developing deimplementation strategies that will result in broad, sustained uptake. Findings of this study, including the feasibility of our approach to data collection, will be used to inform development of a hybrid effectiveness-implementation trial [27] of continuous $\mathrm{SpO}_{2}$ monitoring deimplementation: the Eliminating Monitor Overuse: pulse oximetry (EMO: $\mathrm{SpO}_{2}$ ) trial. Moreover, using a systematic approach to the study of deimplementation will allow for identification of strategies that are broadly generalizable to efforts to reduce overuse in pediatric hospital settings across a wide range of health conditions.

\section{Methods}

\section{Study setting}

This study will be performed within the Pediatric Research in Inpatient Settings Network (PRIS). PRIS is an independent, hospital-based research network that aims to improve the health of and healthcare delivery to hospitalized children and their families. The 117 member-hospitals range in geographic location, size, staffing models, and care practices. Hospitals are categorized as (1) freestanding children's hospitals (standalone hospitals devoted entirely to the care of children including a full range of pediatric subspecialty services), (b) children's hospitals within hospitals (general medical centers that care mainly for adult patients and include a pediatric department offering a full range of pediatric subspecialty services), and (c) community hospitals (general medical centers that care mainly for adult patients and include a pediatric department offering limited or no pediatric subspecialty services).

\section{Aim 1: measure baseline $\mathrm{SpO}_{2}$ monitoring overuse in bronchiolitis}

We will use rigorous observational methods to test the hypothesis that $\mathrm{SpO}_{2}$ overuse exceeds $30 \%$ averaged across sites and that there is sufficiently wide variation in overuse rates between sites to distinguish high and low overuse hospitals. This hypothesis is based on quality improvement initiatives focused more generally at reducing a range of overused practices in bronchiolitis [26] and internal estimates from participating hospitals. Our study builds on prior work by evaluating $\mathrm{SpO}_{2}$ monitoring at a diverse range of hospitals and employing a standardized approach to arrive at accurate, generalizable, and reproducible estimates of practice prevalence.

We will perform repeated cross-sectional observational sampling of $\mathrm{SpO}_{2}$ monitor use among 60 children at each site who are hospitalized during peak bronchiolitis season (December 2018 through March 2019). Eligible patients include children 8 weeks through 23 months old with a primary diagnosis of bronchiolitis in their most recent physician progress note, who are not requiring supplemental oxygen or "room air flow" of $21 \%$ oxygen administered via nasal cannula at any flow rate while hospitalized on non-ICU wards. Patients will be excluded if any of the following criteria are present: $<28$ weeks completed gestation, cyanotic congenital heart disease, pulmonary hypertension, home oxygen requirement, neuromuscular disease, immunodeficiency, and cancer.

Data collection will be operationalized at sites during periodic daytime (between $10 \mathrm{AM}$ and $4 \mathrm{PM}$ ) and nighttime (between 11 PM and 7 AM) in-person observational "rounds." Investigators will aim to collect $50 \%$ of their total number of observations during daytime rounds and $50 \%$ during nighttime rounds. During a data collection round, site investigators will identify all patients currently admitted to non-ICU pediatric wards with bronchiolitis by reviewing the census of each unit that cares for children with bronchiolitis and examining the charts of patients meeting age criteria to determine eligibility. They will then walk to the bedside of each eligible infant with bronchiolitis to confirm that supplemental oxygen and room air flow are off and determine the current continuous monitoring status $\left(\mathrm{SpO}_{2}\right.$, electrocardiographic, neither, both). Site investigators will transmit data using REDCap, a secure research data management tool [28]. A draft data collection form is provided (Additional file 1).

The primary outcome is the overall rate of $\mathrm{SpO}_{2}$ monitoring overuse of patients with bronchiolitis within each hospital, defined as the number of continuously $\mathrm{SpO}_{2}$-monitored patients with bronchiolitis who are not requiring supplemental oxygen or room air flow divided by the total 
number of patients with bronchiolitis who are not requiring supplemental oxygen or room air flow. This study will also measure the following secondary outcomes: (1) use of intermittent $\mathrm{SpO}_{2}$ measurement, (2) use of continuous electrocardiographic monitoring (to explore potential co-occurrence of monitor overuse), and (3) the association between orders and actual monitoring. Overuse rate rankings by hospital type will be generated. We will explore differences in overuse between day and night, race/ethnicity, hospital type, and co-occurrence of electrocardiographic monitoring overuse.

\section{Feasibility}

In terms of statistical power, engaging 38 sites (allowing for $\sim 20 \%$ attrition) will enable us to achieve an acceptable range of low and high overuse hospitals within each hospital type. We determined sample size based on the width of the $95 \%$ confidence interval of the point estimate of the site-level overuse rate. The intent is to have a narrow enough width to separate the lowest overuse hospitals (projected $0-30 \%$ overuse) from the highest overuse hospitals (projected $70-90 \%$ overuse). We hypothesize that a total sample size of 60 patients per hospital will allow for an appropriate balance between data collection feasibility and precision of the point estimates (see Table 1) in this pilot study.

Feasibility of our site recruitment and Aim 1 data collection approaches are important outcomes of this trial and will inform the final design of the $\mathrm{EMO} \mathrm{SpO}_{2}$ trial, a large scale, cluster randomized type 3 hybrid effectiveness-implementation trial [27]. In terms of recruitment and samples size, we have performed a preliminary power calculation for the subsequent EMO $\mathrm{SpO}_{2}$ trial using established methods for cluster-randomized trials [29]; however, the ultimate sample size of the $\mathrm{EMO} \mathrm{SpO}_{2}$ trial will be derived based on findings of this pilot. The thresholds for evaluating feasibility of the recruitment and engagement strategies of this pilot will be defined as (1) the number of participating PRIS sites that complete 60 observations will be 24 or greater (based on a target of 18 as specified in the preliminary power calculation allowing for $25 \%$ attrition), and (2) the number of participating PRIS sites that complete at least $40 \%$ of observations overnight will be 24 or greater. Not attaining these thresholds will trigger design changes to the $\mathrm{EMO} \mathrm{SpO}_{2}$ trial.

Evaluating the association between monitoring orders and observationally obtained data about monitoring, one

Table 1 95\% confidence intervals of overuse estimates across a range of within-hospital patient sample sizes and overuse rates

\begin{tabular}{llll}
\hline & $n=20$ & $n=60$ & $n=100$ \\
\hline $30 \%$ overuse & $11.9-54.3 \%$ & $18.8-43.2 \%$ & $21.2-40.0 \%$ \\
$70 \%$ overuse & $45.7-88.1 \%$ & $56.8-81.2 \%$ & $60.0-78.8 \%$ \\
\hline
\end{tabular}

of the secondary outcomes of this Aim, is important in establishing the validity and feasibility of the data collection protocols for the $\mathrm{EMO} \mathrm{SpO}_{2}$ trial. This pilot will employ a personnel-intensive observational approach to determine which patients are continuously monitored. We hypothesize that this approach is feasible and will be more accurate than examining orders to determine true continuous monitoring status across a wide range of hospitals with varying chart documentation and physiologic monitor data infrastructures. We will determine sensitivity, specificity, and positive and negative predictive values for the relationship between the presence of an order for continuous $\mathrm{SpO}_{2}$ monitoring and actual monitoring status to inform monitoring in the EMO $\mathrm{SpO}_{2}$ trial, overall and within individual hospitals. If the presence of a monitoring order exceeds $85 \%$ for sensitivity and specificity, either overall or in specific sites, we will consider using the order as an accurate indicator for monitoring in the subsequent trial.

\section{Aim 2: identify barriers and facilitators to deimplementing $\mathrm{SpO}_{2}$ monitoring in bronchiolitis}

We will perform semi-structured qualitative interviews with key stakeholders at hospitals with the highest and lowest rates of $\mathrm{SpO}_{2}$ overuse to explore clinical, political, interpersonal, and normative factors surrounding pulse oximetry overuse in bronchiolitis. Interviews will also elucidate processes of initiating and discontinuing pulse oximetry. Then, using the Consolidated Framework for Implementation Research (CFIR) as a guiding framework [30], we will identify barriers and facilitators of deimplementing continuous $\mathrm{SpO}_{2}$ monitoring of stable infants who are breathing room air. CFIR is a widely accepted, pragmatic meta-theoretical framework of constructs that influence effective implementation including Intervention Characteristics (for instance, perceptions of the evidence supporting continuous $\mathrm{SpO}_{2}$ monitoring), the Outer Setting (such as parental preferences or institutional policies and protocols), the Inner Setting (for example, unit/department cultural norms related to monitoring), and Characteristics of Individuals (including knowledge of the clinical guidelines or beliefs about risks of harm from brief desaturations). Finally, using an approach derived from the Intervention Mapping methodology [31], we will ask participants to identify key factors and behaviors associated with adoption, implementation, and sustaining intermittent $\mathrm{SpO}_{2}$ measurement instead of using continuous $\mathrm{SpO}_{2}$ monitoring. A draft interview guide is provided (Additional file 2).

With respect to sample size, we will use a purposive sampling strategy to select a sample of an anticipated 75 stakeholders from the two highest overuse and the two lowest overuse hospitals within each hospital type (freestanding children's hospitals, children's hospitals within general hospitals, and community hospitals). We plan to 
conduct approximately 15 interviews with representatives from each stakeholder group (physicians, nurses, respiratory therapists, administrators, parents) until thematic saturation is achieved within each group. If we fail to achieve thematic saturation after 15 interviews, we will make adjustments to the study budget to allow us to perform additional interviews until thematic saturation is achieved. Parent interviews will focus on monitoring preferences [32].

\section{Qualitative analysis using the CFIR implementation framework}

Data management and analysis of interview transcripts will be supported by the use of NVivo software. Both a priori and grounded theory methods [33] will be used in the coding process. The CFIR framework constructs will serve as an initial coding structure (a priori aspect), but we will also allow new concepts to emerge and become part of the coding scheme (inductive aspect). Process maps [34, 35] delineating $\mathrm{SpO}_{2}$ monitoring initiation and discontinuation processes will be created for each hospital based on descriptions of the processes gleaned from the qualitative interviews. Process maps will specifically identify process failures (barriers) that lead to unnecessary monitoring and successful processes (facilitators) that help clinicians avoid unnecessary monitoring.

Initially, team members will complete coding and process maps independently on a common subset of interviews and compare results to assess the reliability and robustness of the coding scheme. Disagreements will be resolved through team discussion. Prior to proceeding with the remainder of the analysis, we will repeat independent coding and measure inter-rater reliability using Cohen's kappa. If an "almost perfect" kappa of 0.81 or higher [36] is achieved, we will largely employ single reviewer coding, with dual coding for $20 \%$ of transcripts. If the kappa is below 0.81, we will dual code all transcripts. Emerging codes, coding challenges, and coding disagreements will be discussed during standing team meetings.
Coding results will result in the identification of key factors that affect the ultimate outcome. These factors will be incorporated into a Qualitative Comparative Analysis (QCA), a mixed methods approach to examine complex combinations of explanatory factors [37]. This analytic approach is useful for identifying "causal complexity," for instance, situations in which factors may only impact outcomes in the presence of other factors [37, 38]. In QCA, factors identified as salient will be used to create a "truth table" in which each hospital is a row and each identified factor is a column [39, 40]. Cells will indicate the presence or degree of presence of each factor at each hospital, allowing for identification of combinations of factors that appear necessary to achieve the targeted deimplementation outcomes.

\section{Aim 3: development of a multifaceted deimplementation strategy}

Using the CFIR constructs and elements of the Theory of Planned Behavior (TPB) [41], barriers and facilitators identified in our qualitative analysis will be categorized and matched with potential strategies for deimplementation. Potential strategies will be drawn from our stakeholder interviews, Expert Recommendations for Implementing Change (ERIC) strategies [42], the broader implementation science literature on behavior change, and the literature on deimplementation of electrocardiographic monitoring in hospitalized adults [43-50]. Leveraging these implementation science and behavioral science theories and recommendations (CFIR, Theory of Planned Behavior, and ERIC strategies), we will construct a table that serves as a "crosswalk" between the identified barriers or facilitators and potential deimplementation strategies (e.g., Table 2).

Potential strategies (adapted to specifically address deimplementation of continuous $\mathrm{SpO}_{2}$ monitoring overuse) with supporting evidence-based literature will be presented to a Strategy Development Panel focus group and refined based on panel input. The panel will be comprised of 12 of the interviewed stakeholders from high overuse hospitals including at least two physicians, nurses, respiratory

Table 2 Examples of matching barriers that may be identified in Aim 2 to deimplementation strategies

\begin{tabular}{|c|c|c|c|c|c|c|}
\hline $\begin{array}{l}\text { Examples of potential barriers } \\
\text { to deimplementation }\end{array}$ & $\rightarrow$ & $\begin{array}{l}\text { Consolidated Framework for } \\
\text { Implementation Research } \\
\text { constructs [30] }\end{array}$ & $\rightarrow$ & $\begin{array}{l}\text { Elements of } \\
\text { adapted Theory of } \\
\text { Planned Behavior [51] }\end{array}$ & $\rightarrow$ & $\begin{array}{l}\text { Expert Recommendations for } \\
\text { Implementing Change strategy } \\
\text { types [42] }\end{array}$ \\
\hline $\begin{array}{l}\text { Belief that continuous monitoring } \\
\text { is a safety net }\end{array}$ & $\rightarrow$ & $\begin{array}{l}\text { Characteristics of individuals: } \\
\text { knowledge and beliefs }\end{array}$ & $\rightarrow$ & $\begin{array}{l}\text { Behavioral beliefs } \\
\text { contributing to } \\
\text { attitudes }\end{array}$ & $\rightarrow$ & $\begin{array}{l}\text { Educational outreach visits, } \\
\text { build a coalition, learning } \\
\text { collaborative }\end{array}$ \\
\hline $\begin{array}{l}\text { Reluctance to change comfortably } \\
\text { entrenched practice of continuously } \\
\text { monitoring all infants }\end{array}$ & $\rightarrow$ & $\begin{array}{l}\text { Characteristics of individuals: } \\
\text { self-efficacy, Inner setting: } \\
\text { implementation climate }\end{array}$ & $\rightarrow$ & $\begin{array}{l}\text { Self-efficacy, } \\
\text { organizational culture } \\
\text { and climate, habit }\end{array}$ & $\rightarrow$ & $\begin{array}{l}\text { Provide reminders (clinical } \\
\text { decision support), audit and } \\
\text { feedback, clinical champions, } \\
\text { learning collaborative }\end{array}$ \\
\hline $\begin{array}{l}\text { Monitoring because of perceived } \\
\text { parental preference that infant be } \\
\text { monitored (preference may be real } \\
\text { or assumed) }\end{array}$ & $\rightarrow$ & $\begin{array}{l}\text { Characteristics of individuals: } \\
\text { knowledge and beliefs, Outer } \\
\text { setting: patient needs }\end{array}$ & $\rightarrow$ & $\begin{array}{l}\text { Behavioral beliefs } \\
\text { contributing to } \\
\text { attitudes, self-efficacy }\end{array}$ & $\rightarrow$ & $\begin{array}{l}\text { Educational outreach visits, } \\
\text { clinical champions, involving } \\
\text { parents as active participants } \\
\text { in deimplementation effort }\end{array}$ \\
\hline
\end{tabular}


therapists, administrators, and parents. After presenting each potential strategy and the barriers/facilitators it is meant to address, we will use open-ended questions to inquire about the feasibility, acceptability, and anticipated impact of each potential strategy. For example, if a potential strategy involves building clinical decision support alerts into the electronic health record, we will evaluate feasibility by inquiring about the information technology capacity to develop new electronic health record alerts; the timeline for development; and their history of successfully developing, implementing, and evaluating alerts for hospitalized children. The facilitator will apply selected methods from nominal group technique [52] in order to ensure that all members of the panel are able to meaningfully contribute. This will include (1) a group sample size of approximately 12 panelists; (2) allowing a period of time after the potential strategy is introduced for each panelist to write down their initial impressions and ideas; (3) polling each group member, one at a time, until every idea is recorded; and (4) permitting time for discussion, clarification, and modification of each idea.

Detailed specifications [53] of the ten highest impact strategies, as determined by the Strategy Development Panel, will be distributed to all stakeholders previously interviewed from high overuse hospitals $(n=30)$ and site investigators from ten additional hospitals that had overuse rates greater than or equal to the median overuse rate. Using a questionnaire, we will measure stakeholder intentions, attitudes, perceived norms, and self-efficacy/perceived control with respect to each potential strategy and ask stakeholders to rate each potential strategy based on anticipated feasibility, acceptability, and impact. Survey results will be reviewed by the study team and advisory committee, the Strategy Development Panel, and the National Heart, Lung, and Blood Institute Project Scientists to arrive at consensus about the most promising individual strategies to include in a multi-component deimplementation strategy.

\section{Discussion}

This protocol outlines the design of a study that will bridge the gap between the guidelines and evidence that support deimplementation and the actual process of deimplementing continuous $\mathrm{SpO}_{2}$ monitoring in stable infants and children with bronchiolitis who do not require supplemental oxygen.

Given the prevalence of this practice in spite of scientific and professional consensus about its lack of utility, there is a critical need for systematic evaluation of the barriers and facilitators to deimplementation in order to design more effective approaches to deimplementation. Sustained uptake of EMO $\mathrm{SpO}_{2}$ trial protocols for deimplementation hinges on careful elucidation of the current factors influencing adoption of the evidence practice and work with stakeholders to design specific and targeted strategies that address the issues identified. Only with an understanding of the contextual factors contributing to the current practice state will we be able to identify deimplementation strategies with broad feasibility, acceptability, and effectiveness.

In their review, "Letting Go: Conceptualizing intervention de-implementation in public health and social service settings," McKay et al. describe the process of deimplementation as consisting of identifying targets for deimplementation, assessing the context in which deimplementation is to occur, putting into place strategies to support deimplementation practices, and evaluating outcomes of deimplementation efforts [54]. The second step, contextual analysis of barriers and facilitators to a specific deimplementation practice, is a crucial but often overlooked element of successful deimplementation [54]. By focusing on this aspect of deimplementation, our protocol will serve to not only increase the likelihood of successful deimplementation of this specific practice (continuous $\mathrm{SpO}_{2}$ monitoring in stable children with bronchiolitis) but will also add generalizable knowledge about how to conduct systematic contextual analysis as it relates to deimplementation and enhance our understanding of the factors contributing to successful deimplementation.

There are a few potential threats and limitations to successful execution of this study. One potential threat is lower or more homogeneous than anticipated rates of $\mathrm{SpO}_{2}$ overuse. Though this seems unlikely given internal estimates and recent community hospital data, we could consider unit-based (as opposed to hospital based) analysis or apply a historic focus to qualitative analysis, evaluating prior barriers, facilitators, and best practices that led to successful deimplementation. Another potential limitation would be if the Strategy Development Panel determines that deimplementation strategies should be tailored for individual hospitals or hospital types; we will present promising strategies as a "menu" of potential options rather than as a unified, uniform approach.

This multi-site study will produce accurate rates of continuous $\mathrm{SpO}_{2}$ monitoring overuse among stable infants with bronchiolitis; detailed analysis of factors that perpetuate and disrupt overuse; and consensus-derived, theory driven strategies for addressing this prevalent clinical issue. Strengths of this approach include our diverse sample of hospital and stakeholders, which will allow for rich contextual analysis, and the application of multiple implementation science frameworks to the nascent field of deimplementation. As the field of pediatric hospital medicine evolves, it is crucial to develop sound methodologies for implementation and deimplementation to facilitate consistent delivery of high-quality inpatient care. 


\section{Additional files}

Additional file 1: This is a draft of the data collection form proposed for use in Specific Aim 1. (PDF $44 \mathrm{~kb}$ )

Additional file 2: This is a draft of the interview guide proposed for use when interviewing physicians from high overuse hospitals in Specific Aim 2. (PDF $142 \mathrm{~kb}$ )

\section{Abbreviations}

AAP: American Academy of Pediatrics; CFIR: Consolidated Framework for Implementation Research; EMO: $\mathrm{SpO}_{2}$ : Eliminating Monitor Overuse: pulse oximetry; ERIC: Expert Recommendations for Implementing Change; PRIS: Pediatric Research in Inpatient Settings Network; QCA: Qualitative Comparative Analysis; TPB: Theory of Planned Behavior

\section{Acknowledgements}

We thank the PRIS site leads, site principal investigators, and pediatric hospitalists who supported the grant application and have agreed to contribute data to the study.

\section{Funding}

Dr. Rasooly's effort was supported by the Pediatric Hospital Epidemiology and Outcomes Training (PHEOT) Program at The Children's Hospital of Philadelphia, funded by Institutional Research Training Grant 5T32HD060550-09 from the Eunice Kennedy Shriver National Institute of Child Health and Human Development of the National Institutes of Health. This study is supported by Cooperative Agreement 1U01HL143475-01 from the National Heart, Lung, and Blood Institute of the National Institutes of Health (Bonafide, PI). Dr. Bonafide's effort developing this protocol was supported by Career Development Award 5K23HL116427-04 from the National Heart, Lung, and Blood Institute of the National Institutes of Health (Bonafide, PI). Dr. Wolk is an investigator with the Implementation Research Institute (IRI), at the George Warren Brown School of Social Work, Washington University in St. Louis, through an award from the National Institute of Mental Health (5R25MH08091607) and the Department of Veterans Affairs, Health Services Research \& Development Service, Quality Enhancement Research Initiative (QUERI).

The funding bodies played no role in designing the study protocol or writing the manuscript.

\section{Availability of data and materials}

Not applicable

\section{Authors' contributions}

$\mathrm{CPB}, \mathrm{RB}, \mathrm{CBW}, \mathrm{FB}, \mathrm{CPL}, \mathrm{AS}, \mathrm{PWB}$, and $L M M$ designed the study. CPB developed the grant application upon which this manuscript is based. IRR conceived of the presentation of the grant proposal as a study protocol manuscript and drafted the manuscript. All authors critically revised the manuscript for important intellectual content and gave final approval of the version submitted for publication.

\section{Ethics approval and consent to participate}

Research activities described in Specific Aim 1 have been approved by the Children's Hospital of Philadelphia Institutional Review Board (Federal Wide Assurance \# 0000459) with a waiver of consent/parental permission per 45 CFR 46.116(d), a waiver of assent per 45 CFR 46.408(a), and a waiver of HIPAA authorization per 45 CFR 164.512(i)(2)(ii) for recruitment purposes and for main study procedures. Research activities described in Specific Aims 2 and 3 are currently under review at the Children's Hospital of Philadelphia Institutional Review Board (Federal Wide Assurance \# 0000459). We will obtain informed consent from all participants. We have requested a waiver of informed consent documentation under 45CFR46.117(c)(1)(ii) because most sessions will take place by telephone.

\section{Consent for publication}

Not applicable

\section{Competing interests}

Dr. Landrigan has been supported in part by the Children's Hospital Association for his work as an Executive Council member of the Pediatric Research in Inpatient Settings (PRIS) network. Dr. Landrigan has consulted with and holds equity in the I-PASS Institute, which seeks to train institutions in best handoff practices and aid in their implementation. In addition, Dr. Landrigan has received monetary awards, honoraria, and travel reimbursement from multiple academic and professional organizations for teaching and consulting on sleep deprivation, physician performance, handoffs, and safety, and has served as an expert witness in cases regarding patient safety and sleep deprivation. The other authors declare that they have no competing interests.

\section{Publisher's Note}

Springer Nature remains neutral with regard to jurisdictional claims in published maps and institutional affiliations.

\section{Author details}

'Section of Hospital Medicine, Children's Hospital of Philadelphia, Buerger Center for Advanced Pediatric Care, 3500 Civic Center Blvd., 11 th Floor, Philadelphia, PA, USA. ${ }^{2}$ Department of Psychiatry, Perelman School of Medicine, University of Pennsylvania, 3535 Market Street, Suite 3015, Philadelphia, PA, USA. ${ }^{3}$ Department of Medical Ethics and Health Policy, Perelman School of Medicine, University of Pennsylvania, Blockley Hall, 423 Guardian Drive, Philadelphia, PA, USA. ${ }^{4}$ Leonard Davis Institute of Health Economics, University of Pennsylvania, 3535 Market Street, Suite 3006 Philadelphia, PA, USA. ${ }^{5}$ Department of Family Medicine and Community Health, Perelman School of Medicine, University of Pennsylvania, Blockley Hall, 423 Guardian Drive, Philadelphia, PA, USA. ${ }^{6}$ Division of General Pediatrics, Department of Pediatrics, Boston Children's Hospital, 300 Longwood Ave, Enders 1, Boston, MA, USA. ${ }^{7}$ Division of Sleep and Circadian Disorders, Departments of Medicine and Neurology, Brigham and Women's Hospital, 75 Francis Street, Boston, MA, USA. ${ }^{8}$ Harvard Medical School, 25 Shattuck St., Boston, MA, USA. ${ }^{9}$ Department of Pediatrics, University of Cincinnati College of Medicine, 3333 Burnet Ave., MLC 9016, Cincinnati, OH, USA. ${ }^{10}$ Division of Hospital Medicine, Cincinnati Children's Hospital Medical Center, 3333 Burnet Ave., MLC 9016, Cincinnati, OH, USA. ${ }^{11}$ James M. Anderson Center for Health Systems Excellence, Cincinnati Children's Hospital Medical Center, 3333 Burnet Ave., MLC 7014, Cincinnati, OH, USA. ${ }^{12}$ Department of Pediatrics, Section of Hospital Medicine, Children's Hospital Colorado, 13123 E. 16th Street, Aurora, CO, USA. ${ }^{13}$ Adult Child Consortium for Outcomes Research and Healthcare Delivery Sciences, University of Colorado School of Medicine, 13001 East 17th Place, Aurora, CO, USA. ${ }^{14}$ Center for Pediatric Clinical Effectiveness, Children's Hospital of Philadelphia, Roberts Center for Pediatric Research, 2716 South Street, Philadelphia, PA, USA.

Received: 5 October 2018 Accepted: 6 May 2019

Published online: 15 May 2019

\section{References}

1. van Bodegom-Vos L, Davidoff F, Marang-van de Mheen PJ. Implementation and de-implementation: two sides of the same coin? BMJ Qual Saf. 2017;26:495-501.

2. Ubel PA, Asch DA. Creating value in health by understanding and overcoming resistance to de-innovation. Health Aff. 2015;34:239-44.

3. Mensah GA. Embracing dissemination and implementation research in cardiac critical care. Glob Heart. 2014;9:363.

4. Chassin MR, Galvin RW, and the National Roundtable on health care quality. The urgent need to improve health care quality: Institute of Medicine National Roundtable on health care quality. JAMA. 1998:280:1000-5.

5. Brownlee S, Chalkidou K, Doust J, Elshaug AG, Glasziou P, Heath I, et al. Evidence for overuse of medical services around the world. Lancet. 2017; 390:156-68

6. Coon ER, Young PC, Quinonez RA, Morgan DJ, Dhruva SS, Schroeder AR Update on pediatric overuse. Pediatrics. 2017;139:e20162797.

7. Keren $\mathrm{R}$, Luan $\mathrm{X}$, Localio $\mathrm{R}$, Hall M, McLeod L, Dai D, et al. Prioritization of comparative effectiveness research topics in hospital pediatrics. Arch Pediatr Adolesc Med. 2012;166:1155-64.

8. Quinonez RA, Garber MD, Schroeder AR, Alverson BK, Nickel W, Goldstein J, et al. Choosing wisely in pediatric hospital medicine: five opportunities for improved healthcare value. J Hosp Med. 2013:8:479-85.

9. Coon ER, Young PC, Quinonez RA, Morgan DJ, Dhruva SS, Schroeder AR. 2017 update on pediatric medical overuse: a review. JAMA Pediatr. 2018;172:482-6.

10. Parikh K, Hall M, Mittal V, Montalbano A, Mussman GM, Morse RB, et al. Establishing benchmarks for the hospitalized care of children with asthma, bronchiolitis, and pneumonia. Pediatrics. 2014;134:555-62. 
11. Schuh S, Babl FE, Dalziel SR, Freedman SB, Macias CG, Stephens D, et al. Practice variation in acute bronchiolitis: a pediatric emergency research networks study. Pediatrics. 2017;140: e20170842.

12. Zaoutis $T$, Localio AR, Leckerman $K$, Saddlemire $S$, Bertoch D, Keren R Prolonged intravenous therapy versus early transition to oral antimicrobial therapy for acute osteomyelitis in children. Pediatrics. 2009;123:636-42.

13. Brady PW, Conway PH, Goudie A. Length of intravenous antibiotic therapy and treatment failure in infants with urinary tract infections. Pediatrics. 2010; 126:196-203.

14. Lewis-de Los Angeles WW, Thurm C, Hersh AL, Shah SS, Smith MJ, Gerber JS, et al. Trends in intravenous antibiotic duration for urinary tract infections in young infants. Pediatrics. 2017;140:e20171021.

15. Schroeder AR, Shen MW, Biondi EA, Bendel-Stenzel M, Chen CN, French J, et al. Bacteraemic urinary tract infection: management and outcomes in young infants. Arch Dis Child. 2016;101:125-30

16. Hasegawa K, Tsugawa Y, Brown DFM, Mansbach JM, Camargo CA. Trends in bronchiolitis hospitalizations in the United States, 2000-2009. Pediatrics. 2013;132:28-36.

17. Ralston SL, Lieberthal AS, Meissner HC, Alverson BK, Baley JE, Gadomski AM, et al. Clinical practice guideline: the diagnosis, management, and prevention of bronchiolitis. Pediatrics. 2014;134:e1474-502.

18. Principi T, Coates AL, Parkin PC, Stephens D, DaSilva Z, Schuh S. Effect of oxygen desaturations on subsequent medical visits in infants discharged from the emergency department with bronchiolitis. JAMA Pediatr. 2016;170:602-8.

19. McCulloh R, Koster M, Ralston S, Johnson M, Hill V, Koehn K, et al. Use of intermittent vs continuous pulse oximetry for nonhypoxemic infants and young children hospitalized for bronchiolitis: a randomized clinical trial. JAMA Pediatr. 2015:169:898-904.

20. Schuh S, Freedman S, Coates A, Allen U, Parkin PC, Stephens D, et al. Effect of oximetry on hospitalization in bronchiolitis: a randomized clinical trial. JAMA. 2014;312:712-8.

21. Paine CW, Goel W, Ely E, Stave CD, Stemler S, Zander M, et al. Systematic review of physiologic monitor alarm characteristics and pragmatic interventions to reduce alarm frequency. J Hosp Med. 2016;11:136-44.

22. Winters BD, Cvach MM, Bonafide CP, Hu X, Konkani A, O'Connor MF, et al. Technological distractions (part 2): a summary of approaches to manage clinical alarms with intent to reduce alarm fatigue. Crit Care Med. 2018;46:130-7.

23. Bonafide CP, Lin R, Zander M, Graham CS, Paine CW, Rock W, et al. Association between exposure to nonactionable physiologic monitor alarms and response time in a children's hospital. J Hosp Med. 2015;10:345-51.

24. Bonafide CP, Localio AR, Holmes JH, Nadkarni VM, Stemler S, MacMurchy M, et al. Video analysis of factors associated with response time to physiologic monitor alarms in a children's hospital. JAMA Pediatr. 2017;171:524-31.

25. Schondelmeyer AC, Simmons JM, Statile AM, Hofacer KE, Smith R, Prine L, et al. Using quality improvement to reduce continuous pulse oximetry use in children with wheezing. Pediatrics. 2015;135:e1044-51.

26. Ralston SL, Garber MD, Rice-Conboy E, Mussman GM, Shadman KA, Walley $\mathrm{SC}$, et al. A multicenter collaborative to reduce unnecessary care in inpatient bronchiolitis. Pediatrics. 2016;137:e20150851.

27. Curran GM, Bauer M, Mittman B, Pyne JM, Stetler C. Effectivenessimplementation hybrid designs: combining elements of clinical effectiveness and implementation research to enhance public health impact. Med Care. 2012:50:217-26.

28. Harris PA, Taylor R, Thielke R, Payne J, Gonzalez N, Conde JG. Research electronic data capture (REDCap) — a metadata-driven methodology and workflow process for providing translational research informatics support. J Biomed Inform. 2009:42:377-81

29. Shiffman S, Stone AA, Hufford MR. Ecological momentary assessment. Annu Rev Clin Psychol. 2008:4:1-32.

30. Damschroder LJ, Aron DC, Keith RE, Kirsh SR, Alexander JA, Lowery JC. Fostering implementation of health services research findings into practice: a consolidated framework for advancing implementation science. Implement Sci. 2009:4:50.

31. Bartholomew Eldredge LK, Markham CM, Ruiter RAC, Fernandez ME, Kok G, Parcel GS. Planning health promotion programs: an intervention mapping approach. 4th ed. San Francisco: Jossey-Bass; 2016.

32. Kuper A, Reeves S, Levinson W. An introduction to reading and appraising qualitative research. Bmj. 2008;337:a288.

33. Bradley EH, Curry LA, Devers KJ. Qualitative data analysis for health services research: developing taxonomy, themes, and theory. Health Serv Res. 2007;42:1758-72
34. Trebble TM, Hansi N, Hydes T, Smith MA, Baker M. Process mapping the patient journey: an introduction. BMJ. 2010;341:c4078.

35. Taylor AJ, Randall C. Process mapping: enhancing the implementation of the Liverpool Care Pathway. Int J Palliat Nurs. 2007:13:163-7.

36. Landis JR, Koch GG. The measurement of observer agreement for categorical data. Biometrics. 1977;33:159-74.

37. Cragun D, Pal T, Vadaparampil ST, Baldwin J, Hampel H, DeBate RD. Qualitative comparative analysis: a hybrid method for identifying factors associated with program effectiveness. J Mixed Methods Res. 2016;10:251.

38. Aron DC, Lowery J, Tseng C, Conlin P, Kahwati L. De-implementation of inappropriately tight control (of hypoglycemia) for health: protocol with an example of a research grant application. Implement Sci. 2014;9:58.

39. Kane $H$, Lewis MA, Williams PA, Kahwati LC. Using qualitative comparative analysis to understand and quantify translation and implementation. Transl Behav Med. 2014:4:201-8.

40. Forman-Hoffman VL, Middleton JC, McKeeman JL, Stambaugh LF, Christian RB, Gaynes BN, et al. Quality improvement, implementation, and dissemination strategies to improve mental health care for children and adolescents: a systematic review. Implement Sci. 2017;12:93.

41. Ajzen I. The theory of planned behavior. Organ Behav Hum Decis Process. 1991;50:179-211.

42. Powell BJ, Waltz TJ, Chinman MJ, Damschroder LJ, Smith JL, Matthieu MM, et al. A refined compilation of implementation strategies: results from the Expert Recommendations for Implementing Change (ERIC) project. Implement Sci. 2015;10:21.

43. Funk M, Fennie KP, Stephens KE, May JL, Winkler CG, Drew BJ, et al. Association of implementation of practice standards for electrocardiographic monitoring with nurses' knowledge, quality of care, and patient outcomes: findings from the Practical Use of the Latest Standards of Electrocardiography (PULSE) trial. Circ Cardiovasc Qual Outcomes. 2017;10:e003132.

44. Kanwar M, Fares R, Minnick S, Rosman HS, Saravolatz L. Inpatient cardiac telemetry monitoring: are we overdoing it? JCOM. 2008;15:16-20.

45. Leighton $\mathrm{H}$, Kianfar $\mathrm{H}$, Serynek S, Kerwin T. Effect of an electronic ordering system on adherence to the American College of Cardiology/American Heart Association guidelines for cardiac monitoring. Crit Pathw Cardiol. 2013;12:6-8.

46. Benjamin EM, Klugman RA, Luckmann R, Fairchild DG, Abookire SA. Impact of cardiac telemetry on patient safety and cost. Am J Manag Care. 2013;19: e225-32.

47. Dressler R, Dryer MM, Coletti C, Mahoney D, Doorey AJ. Altering overuse of cardiac telemetry in non-intensive care unit settings by hardwiring the use of American Heart Association guidelines. JAMA Intern Med. 2014;174:1852-4.

48. Kansara P, Jackson K, Dressler $R$, et al. Potential of missing life-threatening arrhythmias after limiting the use of cardiac telemetry. JAMA Intern Med. 2015;175:1416-8.

49. Rayo MF, Mansfield J, Eiferman D, Mignery T, White S, Moffatt-Bruce SD. Implementing an institution-wide quality improvement policy to ensure appropriate use of continuous cardiac monitoring: a mixed-methods retrospective data analysis and direct observation study. BMJ Qual Saf. 2016; 25:796-802.

50. Cantillon DJ, Loy M, Burkle A, Pengel S, Brosovich D, Hamilton A, et al. Association between off-site central monitoring using standardized cardiac telemetry and clinical outcomes among non-critically ill patients. JAMA. 2016:316:519-24.

51. Mandell D. Building causal models to explain implementation and exnovation. Presentation at: De-Implementation Scientific Challenges and Prospects Workshop. Bethesda: NHLBI Center for Translation Research and Implementation Science; 2017.

52. Jones J, Hunter D. Consensus methods for medical and health services research. BMJ. 1995;311:376-80.

53. Proctor EK, Powell BJ, McMillen JC. Implementation strategies: recommendations for specifying and reporting. Implement Sci. 2013;8:139.

54. McKay VR, Morshed AB, Brownson RC, Proctor EK, Prusaczyk B. Letting go: conceptualizing intervention de-implementation in public health and social service settings. Am J Community Psychol. 2018;62(1):189-202. 